\title{
COMPARISON OF DIFFERENT VOXEL SIZES OF CONE BEAM COMPUTED TOMOGRAPHY IN ASSESSMENT OF VERTICAL ROOT FRACTURE - AN IN VITRO ANALYSIS
}

\author{
Maha M. Abd Elsattar*, Samah H. Elmeadawy**, Ahmed A. Elsouda*** and Wael M. Safwat ${ }^{* * * *}$
}

\begin{abstract}
Aim: This study aimed to assess the validity of CBCT in the detection of VRF and its orientation in human extracted teeth and to analyze the effect of gutta-percha (GP) endodontic obturation. Finally, to compare different CBCT voxel sizes in VRF diagnosis in both GP obuturated and nonobturated teeth.
\end{abstract}

Materials and Methods: Sixty human teeth were divided into: group I included 30 endodontically treated and GP obturated teeth, group II include 30 endodontically treated but not obturated teeth. Then, VRFs were mechanically induced in all teeth and the teeth were scanned with i-CAT CBCT machine with different voxel sizes of $0.125,0.2,0.25 \mathrm{~mm}$. Then, the teeth were inspected by stereomicroscope to confirm the presence of fracture and to detect the orientation of the fractures to act as the gold standard.

Result: Different CBCT voxel sizes of $0.125,0.2 \& 0.25 \mathrm{~mm}$ were found to have the same validity in the VRF diagnosis with $100 \%$ sensitivity, $96.4 \%$ accuracy and $50 \%$ specificity. There was no significant difference between GP obturated and non obturated groups. VRFs occurred mostly in the bucco-lingual direction.

Conclusions: VRF detection and its orientation can be accurately assessed by different CBCT voxel sizes imaging, thus using CBCT 0.25 voxel with less radiation dose is preferred. The presence of GP did not hinder the VRF orientation.

KEYWORDS: CBCT, vertical root fracture and different voxel sizes.

\footnotetext{
*B.D.S , Faculty of Dentistry, Mansoura University.

**Associate Professor, Department of Oral Medicine, Periodontology, Oral Diagnosis and Radiology, Faculty of Dentistry Mansoura University.

*** Assistant Professor, Department of Endodontics, Faculty of Dentistry, Mansoura University.

**** Assistant Professor, Department of Oral Medicine,Periodontology, Oral Diagnosis and Radiology, Faculty of Dentistry Mansoura University.
} 


\section{INTRODUCTION}

Vertical root fractures may occur in either teeth with an intact pulp or endodontically treated teeth. ${ }^{(1,2)}$ Fracture lines can be limited to the root apex or involve the entire length of the root ${ }^{(3)}$. To overcome unsuitable management, an early diagnosis is needed. An accurate diagnosis is difficult because the signs and symptoms are often vague or non-specific. ${ }^{(4)}$ Radiographic evaluation is critical for diagnosis; two dimensional conventional radiographs can be useful only when the X-ray beam is parallel to the plane of the fracture. Superimposition of neighboring structures is an important factor that reduces the ability to identify root fractures by conventional radiography. ${ }^{(5,6)}$

Through the use of specific dental software, CBCT can provide the clinician with multi-planar view in all three dimensions. ${ }^{(7)}$ Clinically, the quality of CBCT image and its ability to show numerous features are affected by many variables such as the unit itself, the field of view (FOV), the tube voltage and current, the voxel size or other technical factors. ${ }^{(8,9)}$ The variation of parameters can be according to the diagnostic task, however, no protocol recognized for specific dental diagnostic tasks. As regards to image generation and manipulation in CBCT, selection of voxel size can be done randomly (best guess or accessibility in the equipment). ${ }^{(10,11)}$ However, standardization of these parameters has a direct influence on the patient radiation dose through the scanning because they interfere with the time of scan. The ALARA principle should be respected. ${ }^{(12)}$

The CBCT imaging can offer more precise data for diagnosis of diseases or conditions in endodontics, however, there is a little evidence on the effect of the root canal obturation in VRF diagnosis. ${ }^{(13-15)}$ Thus, it is necessary to assess the influence of the gutta-percha root fillings on the diagnosis of VRF using CBCT.

\section{AIM OF THE STUDY}

a) Evaluate the validity of CBCT in the detection of VRF and its orientation in human extracted teeth.

b) Analyze the effect of GP endodontic obturation on the detection of VRF linear measurements and orientation

c) Compare different CBCT voxel sizes to diagnose VRF in both GP obturated and non obturated teeth.

\section{MATERIAL AND METHODS}

\section{Sample Collection:}

This study was approved by an institutional research board of the faculty of Dentistry, Mansoura University code (17020118).

\section{Inclusion Criteria:}

This in vitro study included sixty single rooted recently extracted unrestored human teeth without any root fractures that had not undergone any root canal treatment. The teeth were collected from the Oral Surgery Department, Faculty of Dentistry, Mansoura University. The teeth were selected irrespective of the patient's age and gender.

The extracted teeth included periodontally diseased hopeless teeth and teeth extracted for orthodontic purpose. The teeth comprising; incisors, canines and premolars. The root length of the included teeth was in the range of 12-16 mm which was measured by using a digital caliper.

\section{Exclusion Criteria:}

The teeth were inspected using stereomicroscope (Philippines, SZ2-ILST, 3L41770) Seed and Tissue Lab, Faculty of Agriculture, Mansoura University, to confirm the absence of vertical root fractures, preexisting cracks, caries or noncarious lesions on the root surfaces at $6.7 \mathrm{x}$ magnification. Teeth with fractures, internal or external resorption, an obliterated root canal, acutely curved roots or anomaly were excluded from the study. 


\section{Sample Preparation and study groups:}

The first step for the sample preparation is the removal of any attached alveolar bone or calculus from all tooth surfaces. Then, the teeth were immersed in $1 \%$ hypochlorite solution overnight to remove the soft tissues organic residues and debris. Then, they were stored in a wet environment (i.e. saline solution) during the study except during induction of root fracture and radiographic scan to prevent dehydration.

All root canals were accessed coronally with a diamond bur. Then each tooth had its root canal prepared by the protaper rotary system (Dentsply Maillefer, Bullaigues, Switzerland) until it was size f4 beyond the apical foramen to weaken the roots. The teeth were then decoronated (i.e. the crowns were cut at the level of the cementoenamel junction (CEJ) to eliminate any bias of enamel fractures using a diamond metal disc. Finally the teeth were randomly coded. The root surfaces were covered with a layer of wax having $0.5 \mathrm{~mm}$ thickness for the ultimate radiological reconstruction of the periodontal ligament space.

\section{Study groups:}

The teeth were separated into two main groups:

Group I (endodontically treated and GP obturated):- It consisted of 30 teeth which were prepared and the root canal filled with root canal material (Gutta Percha Points, Protaper universal, Dentsply Maillefer, Bullaigus, Switzerland) and sealer (Zical, antibacterial ZOE Root Canal Sealant, PROVEST Den Pro) .The Gutta percha group had a passively well-fitting cemented protaper F4 gutta percha cone placed in the canal of the teeth using the single cone technique.

Group II (endodotically treated, but not obturated): - It consisted of 30 teeth with its root canals prepared but without root canal obturation.
All roots were initially mounted in an acrylic resin block of $2 \mathrm{~cm}$ in height and $1.5 \mathrm{~cm}$ in diameter to prevent splitting of the roots. All teeth with acrylic blocks are inserted in an acrylic arch (u-shaped) to simulate the human arch shape and geometry (Fig. 1).

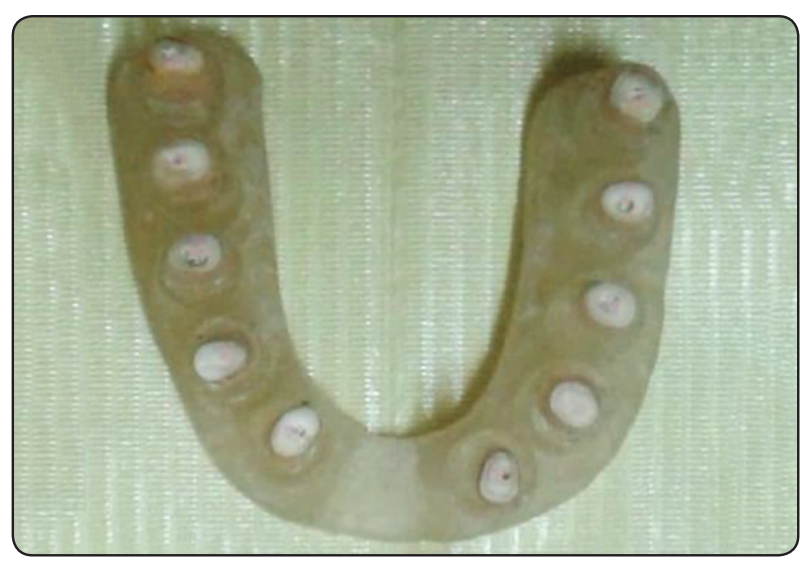

Fig. (1) Teeth with acrylic blocks inside acrylic arches

\section{Vertical root fracture (VRF) induction technique:}

Vertical root fractures were artificially induced by using a tapered plugger with its tip equals $1 \mathrm{~mm}$ in diameter and a hammer. The plugger was inserted into the root canals parallel to the buccal-lingual plane, and a vertical load was applied to it to the apex of the roots by tapping it gently with the hammer. All of the simulated fracture lines initiated from the intracanal portion and extended to the periphery of the root.

Gold standard for determination of VRF was stereomicroscope. After CBCT imaging, all specimens were subsequently detached from the acrylic resin blocks, then marked with $1 \%$ methylene blue dye for staining; after dryness of the roots, the fracture lines were outlined on the root surface, and finally progression of the fracture was inspected again under the stereomicroscope to check the presence and morphology of vertical root fracture at $6.7 \mathrm{x}$ magnification. 
A line having either a vertical or an oblique direction along the root surface with no evident displacement of the fracture segments can be the definition of the fracture line. Only teeth with a non-displaced fracture were accepted in the study. Seven teeth were displaced into separate segments and excluded from the study.

All teeth were coded using 3 point confidence scale rating for the presence or absence of fracture; (0: fracture definitely not present, 1 : questionable fracture, 2: fracture definitely present). The presence of VRF and its orientation were detected by stereomicroscope to act as the gold standard measurements in relation to CBCT assessement. The fractured fragments were attached and fixed to each other by using finger pressure on the outer surfaces of the roots if these segments were associated with a wide separation of the root structure.

\section{Imaging procedures}

Group I before fracture induction and both group I and II after fracture induction were scanned using the CBCT system with «flat-panel image detectors (FPDs»): «i-CAT Next Generation machine» (Imaging science international, ISI, PA, USA). The roots were placed in FOV in a centralized position. The CBCT images were prepared using i-CAT CBCT according to the protocol recommended by the manufacturer set at $120 \mathrm{Kv}$ and $5 \mathrm{~mA}$.

Each root was scanned at a fixed field of view ( $4 \mathrm{~cm}$ height and $16 \mathrm{~cm}$ diameter), with three different voxel sizes $0.125,0.2,0.25$. Before beginning the scan, the CBCT unit was adjusted to obtain a series of homogenous exposures without any object in between the detector and $\mathrm{x}$-ray source. So, dark image offset and linear gain corrections were provided by this procedure. The volume reconstruction was performed with viewer software. The image data sets were recorded to be shown in arbitrary order and raw Dicom data were imported to a $3^{\text {rd }}$ party viewing software (Ondemand 3D App) for a better analysis and measurements.

\section{Image assessment}

Written and verbal directions about CBCT image interpretation with examples of fractured roots in CBCT scans were delivered after a calibration meeting. Assessment of each data set independently by scrolling all axial, sagittal and coronal slices interactively to observe a radiolucent thin line alongside the root surface was done through three blinded observers (one endodontist and two radiologists). Observers were asked to identify the presence or absence of a fracture and its orientation. The viewing time to evaluate the presence or absence of vertical root fractures was unrestricted. Every observer decided presence or absence of the fracture in each data set, i.e. irrespective of in which sectional plane fracture line was seen (yes/no in each root). The observers were first trained on using CBCT software and having access to the raw CBCT data.

The observers recorded their opinion using a 3 point confidence scale rating as follows; 0 : fracture definitely not present, 1: questionable fracture, 2: fracture definitely present. The observers were permitted to click on a location of interest in any one of the planes. The zoom, brightness and contrast guidelines in the software were permitted to be used by the observers to manipulate image characteristics. Examination of a maximum 20 image data sets in a daily session was achieved to avoid visual fatigue. The image datasets were displayed on a 32inch liquid crystal display monitor screen (Samsung LED) with a resolution of $1920 \times 1080$ pixels, in true color (32-bit) in the Ondemand 3D dental imaging software» (Cybermed, Korea). The image datasets were seen two times with at least two week interval between the assessments.

\section{Statistical Analysis of Data}

Data were entered and statistically analyzed using the Statistical Package for Social Sciences (SPSS) version 20.

Validity (Sensitivity, specificity, positive, negative predictive values, and accuracy) were 
calculated using cross tabulation. Reliability of continuous variables were calculated using interclass correlation with strong agreement at $(\mathrm{r}>0.5)$. «p value $\leq 0.05$ » was considered to be statistically significant and all tests were 2 tailed

\section{RESULTS}

In the present study, the intraobserver reliability test of CBCT readings in the $1^{\text {st }}, 2^{\text {nd }}$, and $3^{\text {rd }}$ observers showed that there is a high statistically significant strong positive correlation $(\mathrm{p}=1.000)$ between the three readings of each CBCT voxel size 0.125 , $0.2 \& 0.25 \mathrm{~mm}$. Each voxel size showed perfect correlation with the other 2 readings except CBCT $0.25 \mathrm{~mm}$ voxel size showed non-reliable $3^{\text {rd }}$ readings with the other 2 readings. The interclass correlation between the $1^{\text {st }}$ observer, $2^{\text {nd }}$ and $3^{\text {rd }}$ observer CBCT readings, shows a strong positive correlation between the $1^{\text {st }}, 2^{\text {nd }}$ and $3^{\text {rd }}$ observer CBCT readings in all voxel sizes.

VRFs were assessed by stereomicroscope and CBCT different voxel sizes in endodontically treat- ed obturated (figure 2) and non obturated teeth (figure 3). Table 1 shows the validity of CBCT in VRF diagnosis as compared to stereomicroscope results in group I. CBCT $0.125,0.2$ and $0.25 \mathrm{~mm}$ voxel sizes have the same validity measures in fracture diagnosis as compared to stereomicroscope with $100.0 \%$ sensitivity and negative predictive value, 96.4\% accuracy, $96.3 \%$ positive predictive value with the least measure was specificity $50.0 \%$.

TABLE (1) Validity of CBCT in fracture diagnosis as compared to stereomicroscope results in group I.

\begin{tabular}{|l|c|c|c|}
\hline & $\begin{array}{c}\text { CBCT } \\
\mathbf{0 . 1 2 5}\end{array}$ & $\begin{array}{c}\text { CBCT } \\
\mathbf{0 . 2}\end{array}$ & $\begin{array}{c}\text { CBCT } \\
\mathbf{0 . 2 5}\end{array}$ \\
\hline Sensitivity & $100.0 \%$ & $100.0 \%$ & $100.0 \%$ \\
\hline Specificity & $50.0 \%$ & $50.0 \%$ & $50.0 \%$ \\
\hline Positive predictive value & $96.3 \%$ & $96.3 \%$ & $96.3 \%$ \\
\hline Negative predictive value & $100.0 \%$ & $100.0 \%$ & $100.0 \%$ \\
\hline Accuracy & $96.4 \%$ & $96.4 \%$ & $96.4 \%$ \\
\hline
\end{tabular}
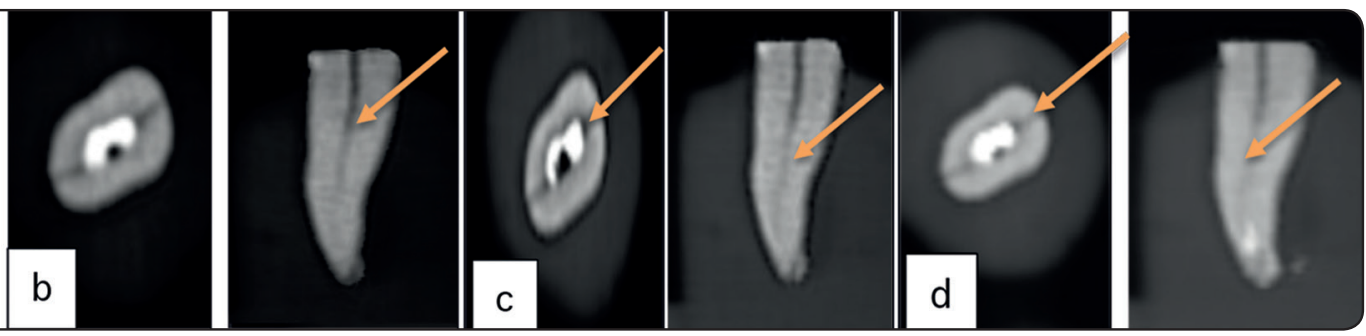

Fig. (2) CBCT scans with three different voxel resolutions, using the i-CAT machine, of an endodontically treated and obturated root after fracture induction. (a): seen by stereomicroscope, (b): axial and sagittal slices at $0.1 \mathrm{~mm}$ voxel size, (c): axial and sagittal slices at $0.2 \mathrm{~mm}$ voxel size, (d): axial and sagittal slices at $0.25 \mathrm{~mm}$ voxel size. Arrow ${ }^{\circ}$ indicates fracture line through the root surface.
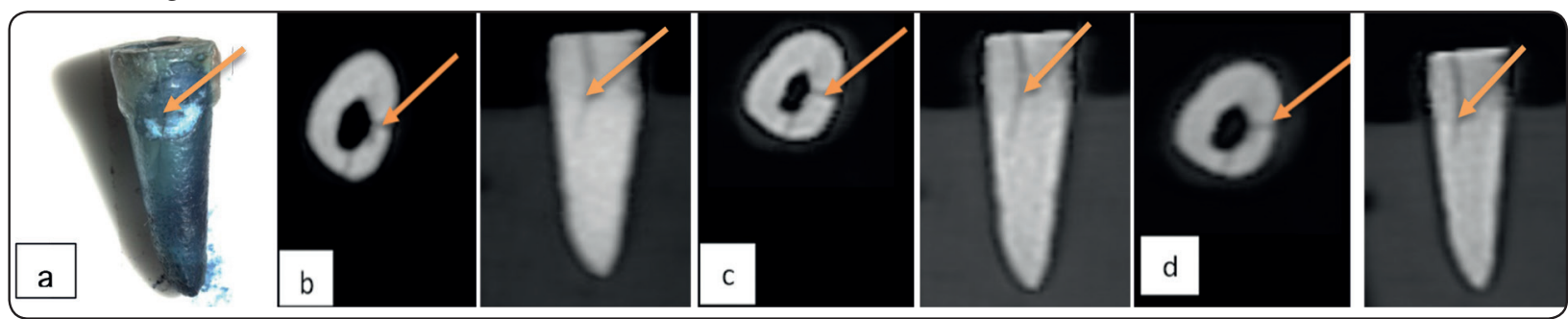

Fig. (3) CBCT scans with three different voxel resolutions, using the i-CAT machine, of an endodontically treated and not obturated root after fracture induction. (a): seen by stereomicroscope, (b): axial and sagittal slices at $0.1 \mathrm{~mm}$ voxel size, (c): axial and sagittal slices at $0.2 \mathrm{~mm}$ voxel size, (d): axial and sagittal slices at $0.25 \mathrm{~mm}$ voxel size. Arrows ${ }^{\circledR}$ indicate fracture line through the root surface. 
Table 2 shows the validity of CBCT in VRF diagnosis as compared to stereomicroscope results in group II. CBCT $0.125,0.2 \& 0.25 \mathrm{~mm}$ voxel sizes had the same validity measures in fracture diagnosis as compared to stereomicroscope with $100.0 \%$ sensitivity and negative predictive value, $96.0 \%$ accuracy, $95.8 \%$ positive predictive value with the least measure was specificity $50.0 \%$. Moreover, group I and II showed the same sensitivity, specificity and negative predictive value, however, group I showed slightly higher positive predictive value and accuracy than group II at all voxel sizes (figure 4).

TABLE (2) Validity of CBCT in fracture diagnosis as compared to stereomicroscope results in group II.

\begin{tabular}{|l|c|c|c|}
\hline & $\begin{array}{c}\text { CBCT } \\
\mathbf{0 . 1 2 5}\end{array}$ & $\begin{array}{c}\text { CBCT } \\
\mathbf{0 . 2}\end{array}$ & $\begin{array}{c}\text { CBCT } \\
\mathbf{0 . 2 5}\end{array}$ \\
\hline Sensitivity & $100.0 \%$ & $100.0 \%$ & $100.0 \%$ \\
\hline Specificity & $50.0 \%$ & $50.0 \%$ & $50.0 \%$ \\
\hline Positive predictive value & $95.8 \%$ & $95.8 \%$ & $95.8 \%$ \\
\hline Negative predictive value & $100.0 \%$ & $100.0 \%$ & $100.0 \%$ \\
\hline Accuracy & $96.0 \%$ & $96.0 \%$ & $96.0 \%$ \\
\hline
\end{tabular}

Table 3 shows the orientation pattern and number of VRFs as detected in the CBCT scan. Almost of all fractures occurred in buccolingual (60.4\%) direction more than in oblique (18.9\%) and mesiodistal (13.2\%) directions.

TABLE (3) Orientation pattern and number of vertical root fractures as detected in the CBCT scan.

\begin{tabular}{c|c|c}
\hline $\begin{array}{c}\text { Direction of } \\
\text { fracture lines }\end{array}$ & $\begin{array}{c}\text { Number of } \\
\text { roots, (\%) }\end{array}$ \\
\hline $\begin{array}{c}\text { Bucco-lingual } \\
\text { direction }\end{array}$ & $32(60.4 \%)$ \\
\hline $\begin{array}{c}\text { Oblique } \\
\text { direction }\end{array}$ & & $10(18.9 \%)$ \\
\hline $\begin{array}{c}\text { Mesio-distal } \\
\text { direction }\end{array}$ & & $7(13.2 \%)$ \\
\hline $\begin{array}{c}\text { Non fractured roots } \\
\text { detected by CBCT }\end{array}$ & & 53 \\
\hline Total & & 5 \\
\hline
\end{tabular}

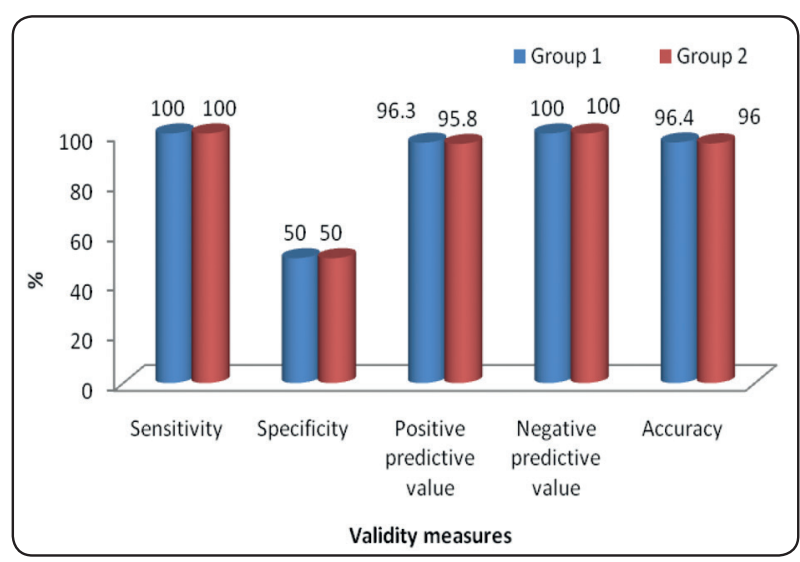

Fig. (4) Teeth with acrylic blocks inside acrylic arches

\section{DISCUSSION}

This study investigated the diagnostic ability of CBCT scans with three different voxel sizes in the detection of VRF linear measurements and orientation in both GP obturated and non obturated teeth. VRFs were induced experimentally by placing a tapered plugger with $1 \mathrm{~mm}$ tip in diameter inside the root canals then tapping it gently with a hammer. This technique was similar to the method that used by Khedmat et al, Melo et al, and Shemesh et al ${ }^{(16-18)}$ As in Jakobson et al and Melo et al, the single gutta-percha cone technique was used in obturation of endodontically treated teeth. ${ }^{(7,19)}$ This was intended to reduce the stresses that can be occurred on the lateral walls of the canal during excessive condensation.

Almost all of the fractures identified by using CBCT were in buccolingual direction more than in mesiodistal and oblique directions, This result is similar to Kambungton et al study. ${ }^{(20)}$ The increased incidence of VRFs in bucco-lingual direction could be explained by the broadest buccolingual than mesiodistal anatomical dimensions of the sample study teeth which mostly consisted of premolars. The morphology of teeth could be a predisposing factor for VRF. Furthermore, mesiodistal and oblique fractures could be distinguished on the CBCT images by all observers. This represents an advantage of 
CBCT over the conventional $\mathrm{x}$-ray in the diagnosis of vertical root fractures.

The observed validity of CBCT in the detection of VRFs was compared to stereomicroscope as the gold standard. The overall sensitivity $(100 \%)$ and accuracy (96.4\% for group I and 96\% for group II) were high and the same for the three voxel resolutions in either GP obturated or non obturated groups. Different studies have detected higher sensitivity of CBCT in detecting VRF in GP and NGP teeth ranging from $98 \%$ to $78 \%$, however, Brady et al ${ }^{(23)}$ have detected lower sensitivity of CBCT $(28 \%)$ in detecting VRF in NGP teeth.

There may be many possible explanations for the increased sensitivity in this study. First, the facility to view the images in the axial plane may be a factor in increasing the sensitivity of the CBCT. Second, this study was in vitro with artificially simulated vertical root fractures. Fractures that occurred after their artificial induction, the separation chances of the fractured margins are larger and its fracture pattern is more obvious than those with natural fractures.

Third, because CBCT scan has three dimensional nature that allows visualization of the fracture line from different angulations and orientations at high contrast. Fourth, there was no superimposed soft tissues or surrounding alveolar bone that might affect the results.

Fifth, the thickness of fractures and the method of VRF induction may also have a direct influence on the CBCT accuracy. ${ }^{(24)}$ The thicker the fracture line, the higher its detectability. The higher sensitivity in this study and study of Hassan et al 2009, $2010^{(22,25)}$, and Ozer 2011 ${ }^{(21)}$ could be explained by the method of VRF induction as all of them used tapered plugger and hammer. This method might lead to incomplete wide fractures that could be detected by CBCT with all voxel sizes. In Melo et al (26) study, which showed a sensitivity of $71 \%$ also used tapered plunger and hammer, but hairline fractured only is included in the study.
The lower sensitivity of $28 \%$ in Brady et al ${ }^{(23)}$ study may be explained by using of a universal testing machine (Instron) for VRF induction. By this technique, these fractures were formed in a highly controlled style because of the gradual increase and continuous monitoring of the force applied to the roots. These induced VRFs may be smaller in width.

Moreover, in this study, the sensitivity of CBCT was higher than specifity (50\% specificity) in all voxels in both groups. Because of low specificity, an intact tooth may be misdiagnosed as a fractured tooth and incorrectly extracted. This result was in accordance with the study of Melo et al ${ }^{(17)}$, who detected that the overall sensitivity of $0.2 \mathrm{~mm}$ voxel size was higher than specificity, and in contrast to the results of other studies. ${ }^{(3,21-23,25,26)}$ The discrepancy observed between these studies might be related to the variance in the sample size or number of observers or study designs.

In this study, no differences were detected between non obturated and GP obturated canals. However, there were cases identified as false positive in readings that identified as VRF but really not fractured as seen by stereomicroscope. This result was coincided with Chen et al, Melo et al, and Hassan et al 2009. ${ }^{(17,22,27)}$ who established that the presence of gutta-percha did not affect the sensitivity of CBCT scans though it decreased the specificity. However, Khedmat et al ${ }^{(16)}$ study who found that gutta-percha apparently decreased the accuracy of CBCT.

The presence of false positive cases could be explained by the nature of the intracanal contents directly influences the diagnostic accuracy of CBCT in detection of vertical root fractures. The presence of hyperdense materials (i.e. gutta-percha and metal post) can lead to severe streaking artifacts. They occur because of extreme beam hardening or photon starvation owing to insufficient photons reaching the detector, resulting in horizontal streaks in the image and noisy projection reconstructions. Beam hardening occurs if the mean energy of the $\mathrm{x}$-ray 
beam is increased due to absorption of lower energy photons more than higher energy photons. This artifact is more marked on CBCT images than conventional computed tomography as the result of the heterochromatic nature of the CBCT $\mathrm{x}$-ray beam and its lower mean kilovolt peak energy. ${ }^{(28)}$

In this study, it was found that there were no significant differences between the validity of different voxel sizes of CBCT in diagnosis of VRFs. Thus, according to the ALARA principle, we can recommend the use of $0.25 \mathrm{~mm}$ voxel resolution as a specific exposure protocol to reduce the patient radiation dose in clinical situations because sharper images with smaller voxel sizes would increase radiation dose to the patient but might afford similar diagnostic outcome as the images with lower resolution. ${ }^{(29)}$

This result was in accordance with da Silveira et al ${ }^{(30)}$ and Ozer ${ }^{(23)}$ studies which did not find significant differences in all voxel sizes ranging from 0.125 to $0.4 \mathrm{~mm}$. On the other hand this result was in contrast with previous study of Melo et al (17) which recommended that $\mathrm{CBCT}$ with high resolution (voxel size smaller than $0.2 \mathrm{~mm}$ ) should be used when root fracture is doubted and not detected in a periapical image.

In the present study, There were 3 of 53 fractured roots could not be diagnosed by CBCT. The explanation was that the diagnostic accuracy of CBCT may depend on the width of $\mathrm{VRF}^{(31)}$, if teeth is obturated or not, radioopacity of filling materials, and milliamperage of $\mathrm{CBCT}{ }^{(32)}$. Because the root fracture prognosis is poor and extraction is often required, false positive or false negative results must be avoided. ${ }^{(17)}$

\section{CONCLUSION}

VRF detection and its orientation can be accurately assessed by different CBCT voxel sizes imaging, thus using CBCT 0.25 voxel with less radiation dose is preferred. The presence of GP did not affect the VRF detection nor its orientation.

\section{REFERENCES}

1. Tamse A, Fuss Z, Lustig J, and Kaplavi J. An evaluation of endodontically treated vertically fractured teeth. J Endod 1999; 25:506-8.

2. Chan CP, Lin CP, Tseng SC, and Jeng JH. Vertical root fracture in endodontically versus non endodontically treated teeth - a survey of 315 cases in Chinese patients. Oral Surg Oral Med Oral Pathol Oral Radiol Endod 1999; 87:504-7.

3. Youssefzadeh S, Gahleitner A, Dorffner R, Bernhart T, and Kainberger FM. Dental vertical root fractures: value of CT in detection, Radiology 1999;210: 545-9.

4. Cohen S, Berman LH, Blanco L, Bakland L, and Kim JS. A demographic analysis of vertical root fractures. Journal of Endodontics 2006;32:1160-3.

5. Kositbowornchai S, Nuansakul R, Sikram S, Sinahawattana $\mathrm{S}$, and Saengmontri S. Root fracture detection: a comparison of direct digital radiography with conventional radiography. Dentomaxillofacial Radiology 2001;30:106-9.

6. Tsesis I, Kamburoglu K, Katz A, Tamse A, Kaffe I, and Kfir A. Comparison of digital with conventional radiography in detection of vertical root fractures in endodontically treated maxillary premolars: an ex vivo study. Oral Surg Oral Med Oral Pathol Oral Radiol Endod 2008;106:124-8.

7. Jakobson SJM, Westphalen VPD, Silva Neto UX, Fariniuk LF, Schroeder AGD, and Carneiro E. The influence of metallic posts in the detection of vertical root fractures using different imaging examinations. Dentomaxillofacial Radiology 2014;43:20130287.

8. Chadwick JW, and Lam EW. The effects of slice thickness and interslice interval on reconstructed cone beam computed tomographic images. Oral Surg Oral Med Oral Pathol Oral Radiol Endod 2010;110: e37-e42.

9. Kamburoglu K, Murat S, Kolsuz E, Kurt H, Yuksel S, and Paksoy C. Comparative assessment of subjective image quality of cross sectional cone-beam computed tomography scans. Journal of Oral Science 2011;53:501-8.

10. Spin-Neto R, Marcantonio J R, Gotfredsen E, and Wenzel A. Exploring CBCT-based DICOM files. A systematic review on the properties of images used to evaluate maxillofacial bone grafts. Journal of Digital Imaging 2011; 24:959-66.

11. Ludlow J B, Davies-Ludlow L E, Brooks S L, and Howerton W B. Dosimetry of 3 CBCT devices for oral and maxillofacial radiology: CB Mercuray, NewTom 3G and i-CAT. Dentomaxillofacial Radiology 2006;35:219-26. 
12. Ballrick JW, Palomo JM, Ruch E, Amberman BD, and Hans MG. Image distortion and spatial resolution of a commercially available cone-beam computed tomography machine. American Journal of Orthodontics and Dentofacial Orthopedics 2008;134:573-82.

13. Queiroz PM, Nascimento HAR, da Paz TDJ, Anacleto FN, and Freitas DQ. Accuracy of digital subtraction radiography in the detection of vertical root fractures. Journal of endodontics 2016;42:896-9.

14. Chang E, Lam E, Shah P, and Azarpazhooh A. Conebeam computed tomography for detecting vertical root fractures in endodontically treated teeth: a systematic review. Journal of endodontics 2016;42:177-85.

15. Rezende Barbosa GL, Sousa Melo SL, Alencar PNB, Nascimento MCC, and Almeida SM. Performance of an artifact reduction algorithm in the diagnosis of in vitro vertical root fracture in four different root filling conditions on CBCT images. International endodontic journal 2016;49: 500-8.

16. Khedmat S, Rouhi N, Drage N, Shokouhinejad N, and Nekoofar MH. Evaluation of three imaging techniques for the detection of vertical root fractures in the absence and presence of gutta-percha root fillings. International endodontic Journal 2012; 45:1004-9.

17. Melo SL, Bortoluzzi E A, Abreu M J, Correa L R, and Correa M. Diagnostic ability of a cone beam computed tomography scan to assess longitudinal root fractures in prosthetically treated teeth. Journal of Endodontics 2010;36:1879-82.

18. Shemesh H, Van Soest G, Wu M K, and Wesselink P R. Diagnosis of vertical root fractures with optical coherence tomography. Journal of Endodontics 2008;34:739-42.

19. Melo SLS, Haiter-Neto F, Correa LR, Scarfe WC and Farman AG. Comparative diagnostic yield of cone beam $\mathrm{CT}$ reconstruction using various software programs on the detection of vertical root fractures. Dentomaxillofacial Radiology 2013;42:20120459.

20. Kambungton J, Janhom A, Prapayasatok S, and Pongsiriwet $\mathrm{S}$. Assessment of vertical root fractures using three modalities: cone beam $\mathrm{CT}$, intraoral digital radiography and film. Dentomaxillofacial Radiology 2012;41:91-5.

21. Ozer SY. Detection of vertical root fractures by using cone beam computed tomography with variable voxel sizes in an In Vitro model. Journal of Endodontics 2011;37:75-9.

22. Hassan B, Metska M E, Ozok A R, van der Stelt P, and Wesselink R. Detection of vertical root fractures in end- odontically treated teeth by a cone beam computed tomography scan. Journal of Endodontics 2009;35:719-22.

23. Brady E, Mannocci F, Brown J, Wilson R, and Patel S. A comparison of cone beam computed tomography and periapical radiography for the detection of vertical root fractures in non endodontically treated teeth. International endodontic journal 2014; 47:735-46.

24. Ozer SY. Detection of vertical root fractures of different thicknesses in endodontically enlarged teeth by cone beam computed tomography versus digital radiography. Journal of Endodontics 2010;36:1245-9.

25. Hassan B, Metska ME, Ozok AR, Stelt PV, and Wesselink PR. Comparison of five cone beam computed tomography systems for the detection of vertical root fractures. Journal of Endodontics 2010;36:126-9.

26. Melo SLS, Haiter-Neto F, Correa LR, Scarfe WC, and Farman A G. Comparative diagnostic yield of cone beam CT reconstruction using various software programs on the detection of vertical root fractures. Dentomaxillofacial Radiology 2013;42:20120459.

27. Chen SC, Chueh LH, Hsiao CK, Wu HP, and Chiang CP. First untoward events and reasons for tooth extraction after nonsurgical endodontic treatment in Taiwan. Journal of Endodontics 2008;34:671-4.

28. Scarfe WC, and Farman AG. What is cone-beam CT and how does it work? Dental Clinics of North America 2008;52:707-30.

29. Davies J, Johnson B, and Drage N. Effective doses from cone beam CT investigation of the jaws. Dentomaxillofacial Radiology 2012;41:30-6.

30. da Silveira PF, Vizzotto MB, Liedke GS, da Silveira HLD, Montagner F, and da Silveira HED. Detection of vertical root fractures by conventional radiographic examination and cone beam computed tomography — an in vitro analysis. Dental Traumatology 2013; 29: 41-6.

31. Makeeva IM, Byakova SF, Novozhilova NE, Adzhieva EK, Golubeva GI, Grachev VI, and Kasatkina IV. Detection of artificially induced vertical root fractures of different widths by cone beam computed tomography in vitro and in vivo. International endodontic journal 2016;49:980-9.

32. Araujo HG, Silva de Souza GQ, Freitas DQ, and de Oliveira-Santos C. Optimization of Tube Current in Conebeam Computed Tomography for the Detection of Vertical Root Fractures with Different Intracanal Materials. J Endod 2017 Oct;43(10):1668-73. 